\title{
Psychological Well-Being and III-Being: Do They Have Distinct or Mirrored Biological Correlates?
}

\author{
Carol D. Ryffa Gayle Dienberg Love ${ }^{a}$ Heather L. Urry ${ }^{a}$ Daniel Muller \\ Melissa A. Rosenkranz ${ }^{a}$ Elliot M. Friedman ${ }^{a}$ Richard J. Davidson ${ }^{a}$ \\ Burton Singer ${ }^{b}$ \\ a University of Wisconsin-Madison, Madison, Wisc., and ${ }^{\mathrm{b}}$ Princeton University, Princeton, N.J., USA
}

\section{Key Words}

Well-being • III-being • Neuroendocrine •

Cardiovascular · Distinct · Mirrored

\begin{abstract}
Background: Increasingly, researchers attend to both positive and negative aspects of mental health. Such distinctions call for clarification of whether psychological well-being and ill-being comprise opposite ends of a bipolar continuum, or are best construed as separate, independent dimensions of mental health. Biology can help resolve this query - bipolarity predicts 'mirrored' biological correlates (i.e. well-being and ill-being correlate similarly with biomarkers, but show opposite directional signs), whereas independence predicts 'distinct' biological correlates (i.e. well-being and ill-being have different biological signatures). Methods: Multiple aspects of psychological well-being (eudaimonic, hedonic) and ill-being (depression, anxiety, anger) were assessed in a sample of aging women $(n=135$, mean age $=74)$ on whom diverse neuroendocrine (salivary cortisol, epinephrine, norepinephrine, DHEA-S) and cardiovascular factors (weight, waist-hip ratio, systolic and diastolic blood pressure, HDL cholesterol, total/HDL cholesterol, glycosylated hemoglobin) were also measured. Results:
\end{abstract}

Measures of psychological well-being and ill-being were significantly linked with numerous biomarkers, with some associations being more strongly evident for respondents aged 75+. Outcomes for seven biomarkers supported the distinct hypothesis, while findings for only two biomarkers supported the mirrored hypothesis. Conclusion: This research adds to the growing literature on how psychological well-being and mental maladjustment are instantiated in biology. Population-based inquiries and challenge studies constitute important future directions.

Copyright (C) 2006 S. Karger AG, Basel

\section{Introduction}

Growing interest in positive psychology [1-4] raises a core question: is well-being merely the flip-side of studying psychological maladjustment, or do well-being and ill-being constitute separate, independent dimensions of mental functioning? The former view sees well-being and ill-being as opposite ends of a bipolar continuum, and thereby suggests that what has been learned about psychological distress and disorder is also definitive for wellbeing. Specifically, those with high levels of ill-being (e.g. depression) would be expected to show low levels of well-

\section{KARGER}

(C) 2006 S. Karger AG, Basel

Fax +41613061234

E-Mail karger@karger.ch

www.karger.com
Accessible online at: www.karger.com/pps 
being (e.g. happiness, life purpose), and vice versa. The independence view, in contrast, asserts that well-being and ill-being are largely distinct domains of mental functioning, such that knowledge about the causes, consequences and correlates of one does not extrapolate to the other.

Research on positive and negative affect was the initial forum for asking questions about bipolarity versus independence, with early findings showing that the two types of affect were largely independent [5]. Although inversely correlated, the association between positive and negative affect was not strong (i.e. being high on one did not predict standing on the other). In addition, the two showed distinct correlates with other variables. This work spawned extensive subsequent inquiry that has continued to probe the bipolarity versus independence of positive and negative affect using quantitative, structural analyses [6-8].

Along the way, other studies documented that the absence of ill-being (e.g. depressive symptoms, or major depression) is no guarantee of possessing high well-being [9, 10]. In fact, some individuals show high levels of both illbeing and well-being, while others are free from major psychological disorders, but lack meaningful life engagement. At the level of neural circuitry, well-being and illbeing have also been shown to be differentially instantiated in the brain $[11,12]$. In the clinical context, it has been observed that the long-term treatment of ill-being (i.e. recurrent depression) is not achieved solely by reducing negative cognition, but also requires promotion of well-being $[13,14]$.

Biology provides further opportunities for assessing whether well-being and ill-being are opposite sides of the same coin, or represent fundamentally distinct aspects of psychological functioning. The single continuum, bipolarity perspective predicts 'mirrored' outcomes (i.e. wellbeing and ill-being have largely similar biological correlates, but with opposite directional signs). In contrast, the independent perspective predicts 'distinct' biological correlates (i.e. well-being and ill-being have different biological signatures). Testing the mirrored versus distinct hypotheses is important for clarifying the extent to which positive psychology is uniquely consequential for understanding mind/body relationships, or is merely the flipside of extensive knowledge on biological underpinnings of mental distress and disorder. If the neurophysiological substrates of well-being [15] are different from those of ill-being, there may also be distinct mechanisms for preventing illness and disease including in contexts of known risk [16].
Psychological ill-being has been extensively linked with biology. For example, depressive symptoms and negative affect have been correlated with elevated cortisol and norepinephrine [17-20] as well as with increased cardiovascular risk [21, 22]. Trait anxiety has been associated with elevated glycosylated hemoglobin, elevated waist-hip ratio and depressed HDL cholesterol [22-24]. Trait anger, in turn, has been associated with greater morning to evening decline in salivary cortisol, elevated blood pressure and increased visceral adipose tissue [2527].

Far fewer investigations have examined links between well-being and biology. High levels of purposeful life engagement (eudaimonic well-being) have been associated with significantly lower levels of cortisol as well as lower cardiovascular risk and musculoskeletal symptoms [28, 29]. Positive relations with others and purpose in life have also been linked with lower levels of inflammatory markers [30]. Similarly, social connectedness has been linked with lower resting blood pressure and lower levels of stress hormones (urinary epinephrine, norepinephrine, cortisol) [31]. And, self-enhancing thoughts have been linked with lower salivary cortisol and lower cardiovascular response to a laboratory stressor [32].

Rarely, if ever, have studies investigated psychological well-being, ill-being and biology simultaneously, thus allowing for direct tests of whether they have distinct or mirrored biological signatures. The present investigation addressed this question in a sample of aging women (aged 61-91) on whom detailed measures of well-being and illbeing were obtained. Multiple assessments of neuroendocrine factors (daily salivary cortisol, epinephrine, norepinephrine, DHEA-S) and cardiovascular risk (weight, waist-hip ratio, systolic and diastolic blood pressure, HDL and total/HDL cholesterol, glycosylated hemoglobin) were also obtained. Because prior research has documented age differences in well-being [33, 34], we also probed whether the biological correlates of well-being and ill-being differed between respondents in early versus later decades of aging.

\section{Methods}

\section{Sample}

The sample consisted of 135 women, all of whom had participated in a prior longitudinal study built around the transition of community relocation. Additional research support allowed for the recruitment of approximately half of the original sample for new data collection that included psychosocial assessments and a comprehensive array of biomarkers. There were no selection cri- 
teria, apart from being able to complete the questionnaires and visit the General Clinical Research Center (GCRC) for biological assessments. The newly recruited sample was not significantly different from the original sample with regard to health (chronic conditions, health symptoms), income, or marital status. However, the biomarker sample was significantly younger and had more education than the original sample. Biomarker respondents also had higher scores on four of six aspects of eudaimonic well-being compared with the original sample. The sample ranged in age from 61 to 91 (mean $=74.0 ; \mathrm{SD}=7.08)$. Respondents had moderate incomes (mean $=$ USD 26,360; SD = USD 18,340) and slightly more than a high school education (mean $=14.1$ years of schooling; $\mathrm{SD}=2.8$ years). Over half $(55.6 \%)$ were widowed, with the rest married $(17 \%)$, never married $(8.9 \%)$, or divorced or separated $(18.5 \%)$.

\section{Well-Being Measures}

Self-administered questionnaires were sent to respondents 3-4 weeks prior to their visit to the UW-Madison campus for the biomarker assessments. These were completed independently and returned to investigators at the time of their campus visit. Consistent with recent formulations [4], well-being was assessed with both eudaimonic and hedonic measures. The former addresses self-development and purposeful life engagement, while the latter focuses on happiness and contentment.

Eudaimonic Well-Being. Active engagement with the existential challenges of living was operationalized with six scales: autonomy, environmental mastery, personal growth, positive relations with others, purpose in life and self-acceptance. These were based on Ryff's [35] theoretical integration of numerous formulations of positive functioning. The hypothesized 6-factor structure of well-being has been supported by data from a national sample of Americans [36]. Recent work has also documented that eudaimonic well-being is empirically distinct from, yet related to hedonic well-being [3]. In this study, each well-being dimension was measured with 14 selfdescriptive items (scale range $=14-84$ ). Cronbach's alpha coefficients for the six scales ranged from 0.85 to 0.91 . In the analysis, all eudaimonic scales were cubed to symmetrize the distribution of scores.

Hedonic Well-Being. Hedonic well-being was assessed with the positive affect scale of the PANAS Inventory [37]. The scale consists of 10 items that gather information about the respondent's affective state (scale range $=10-50$ ). Items for positive affect included feeling interested, excited, strong, enthusiastic, proud, alert, inspired, determined, attentive and active. Respondents were asked to indicate the extent to which the descriptive terms comprising the scale characterize their feelings and emotions on an average day. Hedonic well-being was also assessed with the Positive affect scale of the short-form Mood and Anxiety Symptom Questionnaire (MASQ) [38]. This scale included 14 items (scale range $=14-70$ ) that capture joy-in-living aspects of positive affect (e.g. felt happy, cheerful, optimistic, up, looked forward to things with enjoyment, having a lot of fun). Respondents answered how much they had felt this way during the past week. High scores indicate high positive affect. Alpha coefficients are 0.85 and 0.94 , respectively, for the PANAS and MASQ positive scales. The two scales correlated $0.65(\mathrm{p}<0.001)$ with each other. In the correlational analysis, the MASQ scale was squared to symmetrize the distribution of scores.

\section{Ill-Being Measures}

Psychological ill-being was measured in terms of four different assessments: negative affect, depressive symptoms, trait anxiety and trait anger. These are described below.

Negative Affect. Ten items (feeling distressed, upset, guilty, scared, hostile, irritable, ashamed, nervous, jittery, afraid) from the PANAS Inventory [37] were used to measure negative affect. Respondents indicated the extent to which these terms described their feelings on an average day. High scores indicate high negative affect. The alpha coefficient for these items was 0.89 .

Depressive Symptoms. These were assessed using the CES-D Scale [39], a 20-item instrument (scale range $=0-60$ ). Respondents answered each item with regard to how much they had experienced each symptom over the past week. High scores indicate high depressive symptoms. The alpha coefficient for depressive symptoms was 0.89 .

Trait Anxiety. Ten items were used to operationalize trait anxiety [40]. The scale range was 10-40, with high scores indicating high trait anxiety. The alpha coefficient for this scale was 0.88 . In the correlational analysis, the square root of this score was taken to symmetrize the distribution.

Trait Anger. Ten items were used to operationalize trait anger [40]. The scale range was 10-40, with high scores indicating high trait anger. The alpha coefficient for this scale was 0.92 .

\section{Biological Measures}

Respondents were checked into the GCRC located within the UW Hospital and Clinics for an overnight stay. A trained nurse or physician took the respondent's medical history and conducted a physical health examination. GCRC nursing staff obtained blood samples and 12-hour urine samples. During these visits, respondents were given instructions and supplies for providing the daily saliva samples upon their return home.

Neuroendocrine Function. Biomarkers linked to neuroendocrine function included overnight urinary cortisol, daily salivary cortisol, overnight urinary epinephrine and norepinephrine, and from the fasting blood samples described above, DHEA-S. Urinary cortisol, epinephrine and norepinephrine levels were adjusted for urine creatinine levels. Urinary free cortisol levels were measured via radioimmunoassay at the ARUP Laboratory (Salt Lake City, Utah, USA). Urinary epinephrine and norepinephrine levels were measured via liquid chromatography at the University of Wisconsin Hospital and Clinics Clinical Laboratory. In the statistical analyses, the natural log of epinephrine was taken to symmetrize the distribution, and the square root of the norepinephrine values was taken for the same reason.

Subjects provided saliva samples three times a day for 4 days at home. The first sample was collected in the morning after the subject had been awake for $30 \mathrm{~min}$, but before brushing teeth, drinking coffee or eating breakfast. The second sample was collected at midday, before eating lunch, and the third sample in the evening before brushing teeth and going to bed. Cortisol levels were measured with the Salimetrics (State College, Pa., USA) cortisol enzyme immunoassay kit. Prior to the assay, samples are centrifuged for $10 \mathrm{~min}$ at $5,000 \mathrm{rpm}$ to remove cellular and bacterial debris that are inherent in saliva samples. Assay results were considered acceptable only if the coefficient of variation (CV) for the duplicate measurement of the sample was less than or equal to $20 \%$. The mean inter-assay CV is $7.5 \%$, and the mean intra-assay $\mathrm{CV}$ is $3.8 \%$. The detection limit for this assay is $0.007 \mu \mathrm{g} / \mathrm{dl}$. 
Cardiovascular Function. Biomarkers linked to cardiovascular function included weight, waist-hip ratio, systolic and diastolic blood pressure, HDL and total cholesterol and glycosylated hemoglobin. Waist-hip ratio was calculated on the basis of waist circumference (measured at its narrowest point between the ribs and iliac crest) and hip circumference (measured at the maximal point of the buttocks). Systolic and diastolic blood pressure was calculated as the average of three blood pressure readings after $5 \mathrm{~min}$ of quiet sitting. Fasting blood samples for assays of HDL cholesterol, total cholesterol and glycosylated hemoglobin were obtained before 7:00 AM during the respondents' overnight stay at the GCRC. In the statistical analyses, the square root of weight was taken to symmetrize the distribution, and the natural log of glycoslyated hemoglobin was taken for the same reason.

Medications. Some respondents reported taking medications to treat high blood pressure or high cholesterol. All analyses pertaining to these biomarkers controlled for such medications. Analyses involving salivary cortisol also controlled for whether respondents reported taking any steroidal medications.

\section{Statistical Analyses}

Data analysis was conducted in multiple steps. First, frequency distributions for all measures (psychological and biological) were examined and symmetrized as needed. Such normalizing transformations were noted, where appropriate, in the above description of measures. Second, outliers were Winsorized [41], meaning that extreme observations (i.e. those above the 97 th percentile and below the 3rd percentile) were replaced by the value of the nearest unaffected observation. Third, correlations for the full sample as well as for the age 75+ subsample were calculated between biomarkers and scales of well-being or ill-being. Using a per-comparison error rate, we identified correlations that were significantly different from zero at levels of $0.05,0.01$ and 0.001 . They are reported in tables $1,2$.

Finally, significant coefficients from the above analyses were assembled in a table that clarified overall patterns of support for the distinct versus mirrored hypotheses. In this step, we also tested for significant differences between correlations of well-being or illbeing with the same biomarker. That is, for each significant effect obtained for a specific biomarker and well-being or ill-being, we investigated whether that correlation was significantly different from the parallel correlation for the same biomarker and ill-being or well-being, respectively. Confidence limits for these differences between correlated correlation coefficients, and the corresponding $p$ values, were calculated using equations (1)-(4) in Meng et al. [42]. A multiple comparison adjustment was applied in these analyses. Comparisons that were significant controlling for false discovery rates of 0.05 are presented in table 3 . For a detailed discussion of the false discovery rate and its rationale as a multiple comparison procedure see Benjamini and Hochberg [43].

\section{Results}

Well-Being, Ill-Being and Neuroendocrine Correlates

Table 1 presents bivariate correlations between scales of well-being or ill-being and neuroendocrine measures. Not included in the table are eudaimonic measures of environmental mastery and self-acceptance as well as both hedonic scales of positive affect as no significant effects were evident for these variables. Similarly, no significant effects were found for urinary cortisol, which is also not included in the table. With regard to salivary cortisol, significant positive associations were found between the average daily slope ( 3 assessments per day across 4 days) with personal growth and purpose in life, but only for the aged $75+$ sample $(n=52)$. Directional patterns for these outcomes are clarified below. Epinephrine was positively correlated with positive relations with others, and norepinephrine was positively correlated with autonomy. With regard to ill-being, no significant effects were evident for negative affect or trait anxiety (scales not shown in the table). However, trait anger was positively correlated with epinephrine, and depressive symptoms (CESD) were positively correlated with DHEA-S, but only for respondents aged $75+(n=66)$.

Figures 1 and 2 clarify the direction of effects for the average daily slope of salivary cortisol. The average slope ranged from strongly negative values (steeply downward slopes) to near zero (nearly flat slopes). Well-being scores (cubed to symmetrize distributions) plotted above these values show that those with higher personal growth and purpose in life had flatter daily slopes in salivary cortisol. Examination of the means at the three time points (early morning, mid-day, late evening) further revealed that those with higher personal growth and purpose in life started the day with lower levels of salivary cortisol and stayed lower throughout the day than those with lower levels of growth and purpose.

\section{Well-Being, Ill-Being and Cardiovascular Correlates}

Table 2 presents bivariate correlations between measures of well-being or ill-being with cardiovascular factors. No significant associations were evident for eudaimonic scales of autonomy, environmental mastery, or self-acceptance, or for the PANAS positive affect scale of hedonic well-being (scales not included in the table). Scores on positive relations with others were negatively linked with weight, waist-hip ratio and glycosylated hemoglobin. Positive relations with others remained a negative correlate of waist hip ratio even among those aged $75+$, despite the reduction in sample size. Personal growth was positively correlated with HDL, the 'good' cholesterol, and negatively with the total-to-HDL cholesterol ratio. Purpose in life was also positively correlated with HDL cholesterol and negatively with waist-hip ratio. The MASQ positive affect scale was also positively correlated with HDL cholesterol.
Ryff/Dienberg Love/Urry/Muller/ Rosenkranz/Friedman/Davidson/Singer 
Table 1. Correlations between measures of well-being, ill-being and neuroendocrine factors $(\mathrm{n}=135$, age $=61-91)$

\begin{tabular}{|c|c|c|c|c|c|c|}
\hline \multirow{2}{*}{$\begin{array}{l}\text { Neuroendocrine } \\
\text { factors }\end{array}$} & \multicolumn{4}{|c|}{ Well-being $^{1}$} & \multicolumn{2}{|l|}{ Ill-being } \\
\hline & $\begin{array}{l}\text { positive } \\
\text { relations }\end{array}$ & $\begin{array}{l}\text { personal } \\
\text { growth }\end{array}$ & $\begin{array}{l}\text { purpose } \\
\text { in life }\end{array}$ & $\begin{array}{l}\text { auton- } \\
\text { omy }\end{array}$ & CES-D & $\begin{array}{l}\text { anger } \\
\text { (trait) }\end{array}$ \\
\hline \multicolumn{7}{|c|}{ Salivary cortisol (daily slope) } \\
\hline Age $75+(n=52)$ & 0.10 & $0.29 *$ & $0.29 *$ & -0.04 & -0.16 & -0.20 \\
\hline Epinephrine $^{2}$ & $0.20 *$ & 0.02 & 0.02 & 0.002 & 0.002 & $0.24 * *$ \\
\hline Norepinephrine $^{3}$ & 0.08 & 0.06 & -0.02 & $0.21^{* *}$ & -0.06 & 0.05 \\
\hline \multicolumn{7}{|l|}{ DHEA-S } \\
\hline Age $75+(n=66)$ & -0.13 & -0.08 & -0.05 & -0.08 & $0.26^{*}$ & 0.10 \\
\hline \multicolumn{7}{|c|}{$\begin{array}{l}* \mathrm{p}<0.05, * * \mathrm{p}<0.01 . \text { Effect size }(\mathrm{ES}) \text { ranges: } * 0.40<\mathrm{ES}<0.60 ; * * 0.46<\mathrm{ES}<0.51 \text {. } \\
{ }_{1}^{1} \text { Well-being scales are cubed to symmetrize distributions. } \\
{ }^{2} \text { Natural log taken to symmetrize distribution. } \\
{ }^{3} \text { Square root taken to symmetrize distribution. }\end{array}$} \\
\hline
\end{tabular}

Table 2. Correlations between measures of well-being, ill-being and cardiovascular factors $(\mathrm{n}=135$, age $=$ 61-91)

\begin{tabular}{|c|c|c|c|c|c|c|c|c|}
\hline \multirow[t]{2}{*}{ Cardiovascular factors } & \multicolumn{4}{|c|}{ Well-being ${ }^{1}$} & \multicolumn{4}{|l|}{ Ill-being } \\
\hline & $\begin{array}{l}\text { positive } \\
\text { relations }\end{array}$ & $\begin{array}{l}\text { personal } \\
\text { growth }\end{array}$ & $\begin{array}{l}\text { purpose } \\
\text { in life }\end{array}$ & $\begin{array}{l}\text { positive } \\
\text { affect }^{2}\end{array}$ & $\begin{array}{l}\text { negative } \\
\text { affect }\end{array}$ & CES-D & $\begin{array}{l}\text { anxiety } \\
\text { (trait) }^{3}\end{array}$ & $\begin{array}{l}\text { anger } \\
\text { (trait) }\end{array}$ \\
\hline Weight $^{3}$ & $-0.26 * *$ & -0.07 & -0.15 & 0.01 & 0.03 & $0.22 * *$ & -0.05 & 0.03 \\
\hline Waist-hip ratio & $-0.32 * *$ & -0.15 & $-0.17 *$ & -0.06 & 0.13 & 0.07 & 0.10 & 0.04 \\
\hline Age $75+(n=66)$ & $-0.29 *$ & -0.08 & -0.16 & -0.20 & 0.13 & 0.08 & 0.17 & 0.18 \\
\hline Systolic blood pressure & -0.09 & -0.01 & -0.09 & 0.12 & $-0.17 *$ & -0.06 & -0.15 & $-0.17 *$ \\
\hline Age $75+(n=66)$ & -0.08 & 0.11 & -0.09 & 0.13 & $-0.35^{* *}$ & -0.15 & $-0.32 * *$ & -0.22 \\
\hline HDL cholesterol & 0.16 & $0.18^{*}$ & $0.22 * *$ & $0.18^{*}$ & 0.09 & -0.13 & -0.004 & 0.03 \\
\hline Total/HDL cholesterol & -0.10 & $-0.16^{*}$ & -0.15 & -0.13 & 0.08 & 0.11 & -0.03 & 0.03 \\
\hline Glycosylated $\mathrm{HG}^{4}$ & $-0.21 * *$ & -0.10 & -0.13 & -0.10 & $0.20 *$ & 0.16 & $0.18^{*}$ & $0.25^{* *}$ \\
\hline Age $75+(n=66)$ & -0.14 & -0.20 & -0.15 & -0.11 & 0.23 & 0.17 & 0.16 & $0.41^{* * *}$ \\
\hline $\begin{array}{l}{ }^{*} \mathrm{p}<0.05, * * \mathrm{p}<0.0 \\
\mathrm{ES}|<0.6 ; * * 0.42<| \mathrm{E} \\
1 \text { Well-being scales ar } \\
{ }^{2} \text { MASQ, squared to } \\
{ }^{3} \text { Square root taken } \\
{ }^{4} \text { Natural log taken to }\end{array}$ & $\begin{array}{l}* * * \mathrm{p}<0.0 \\
<0.56 ; * * * \\
\text { cubed to sy } \\
\text { mmetrize d } \\
\text { ymmetrize } \\
\text { ymmetrize }\end{array}$ & $\begin{array}{l}01 . \text { Effect } \\
* 0.60<\mid 1 \\
\text { mmetrize } \\
\text { distributior } \\
\text { distributi } \\
\text { distributic }\end{array}$ & $\begin{array}{l}\text { size }(\mathrm{ES}) \mathrm{rc} \\
\mathrm{ES} \mid<0.70 \\
\text { distributio } \\
\text {. } \\
\text { ons. } \\
\text { n. }\end{array}$ & inges assoc & ated with c & orrespond & ng p value & $\mathrm{s}: * 0.37<$ \\
\hline
\end{tabular}

With regard to ill-being, negative affect (PANAS) was negatively correlated with systolic blood pressure. This effect became notably stronger when the sample was limited to those aged 75+. Depressive symptoms (CES-D) were positively correlated with weight. Trait anxiety was negatively correlated with systolic blood pressure, but only for those aged $75+$. Trait anxiety was also positively correlated with glycosylated hemoglobin. Finally, trait anger was also negatively correlated with systolic blood pressure and positively correlated with glycosylated hemoglobin. The latter finding was strongly evident for the full sample, but was particularly strong for the age $75+$ sample $\left(0.41^{* * *}\right)$.

\section{Overall Support for Distinct versus Mirrored Hypotheses}

Table 3 summarizes the above findings according to those showing support for the distinct versus mirrored 
Fig. 1. Relationship between average slope of daily salivary cortisol and personal growth (older women aged $75+, \mathrm{n}=52$ ).
Fig. 2. Relationship between average slope of daily salivary cortisol and purpose in life (older women aged $75+, \mathrm{n}=52$ ).
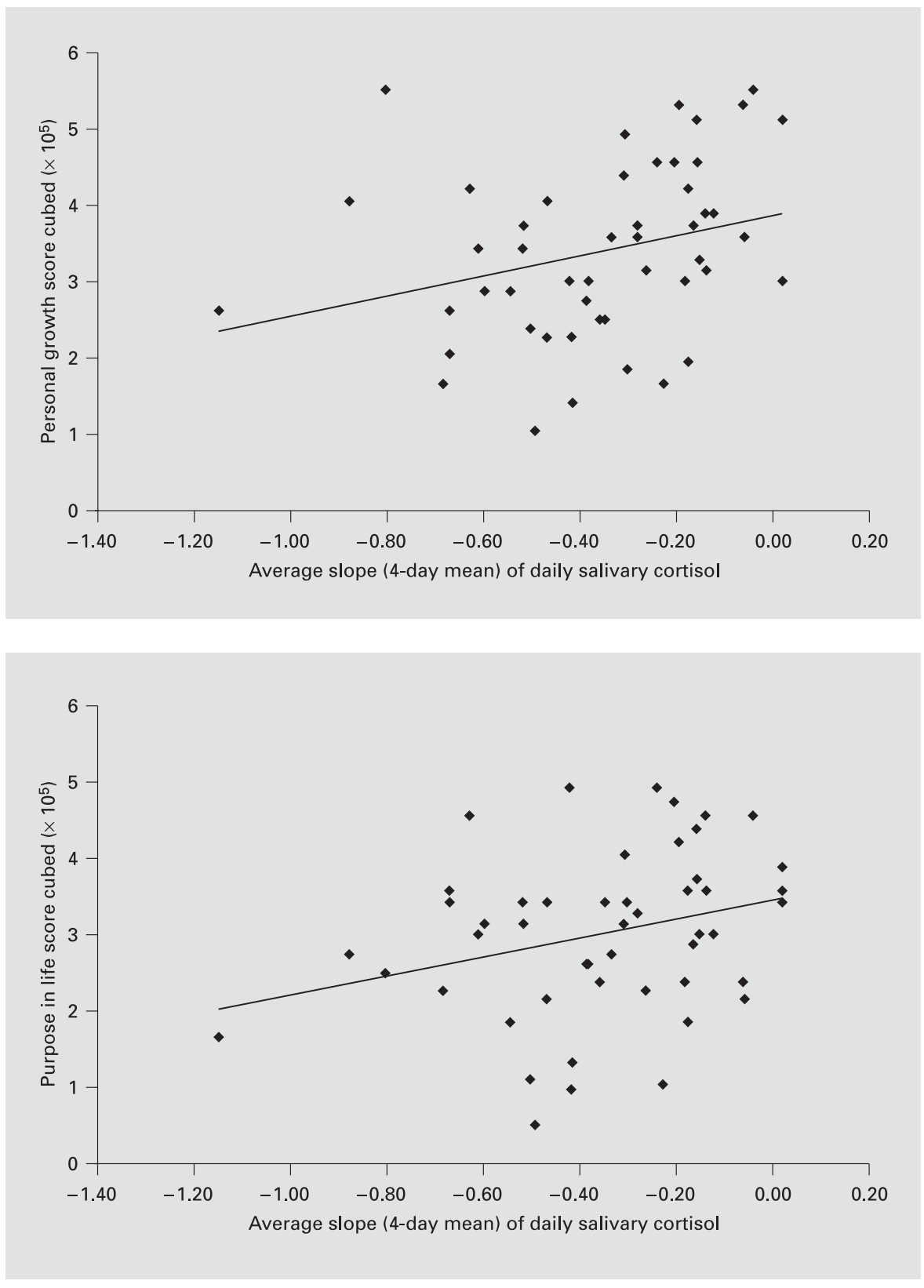

hypotheses. The columns of the table divide the correlations of well-being and biomarkers into those with significant positive association, no significant link, or significant negative associations. The rows of the table divide the correlations of ill-being and biomarkers in the same fashion. The four dark-shaded cells identify outcomes that support the 'distinct' hypothesis - i.e., instances in which a significant association for well-being (or, respectively ill-being) with a specific biomarker was accompanied by a nonsignificant finding for measures of ill-being (or, respectively well-being) and the same biomarker. The two light-shaded cells (upper right, lower left) identify outcomes that support the 'mirrored' hypothesis, i.e. instances in which well-being and ill-being measures were both significantly correlated with the same biomarker, but with opposite directional signs. The two remaining unshaded cells (upper left, lower right) identify outcomes in which well- and ill-being scales correlated in the same direction with the same biomarker. 
Table 3. Ill-being, well-being and biomarkers: distinct or mirrored associational patterns

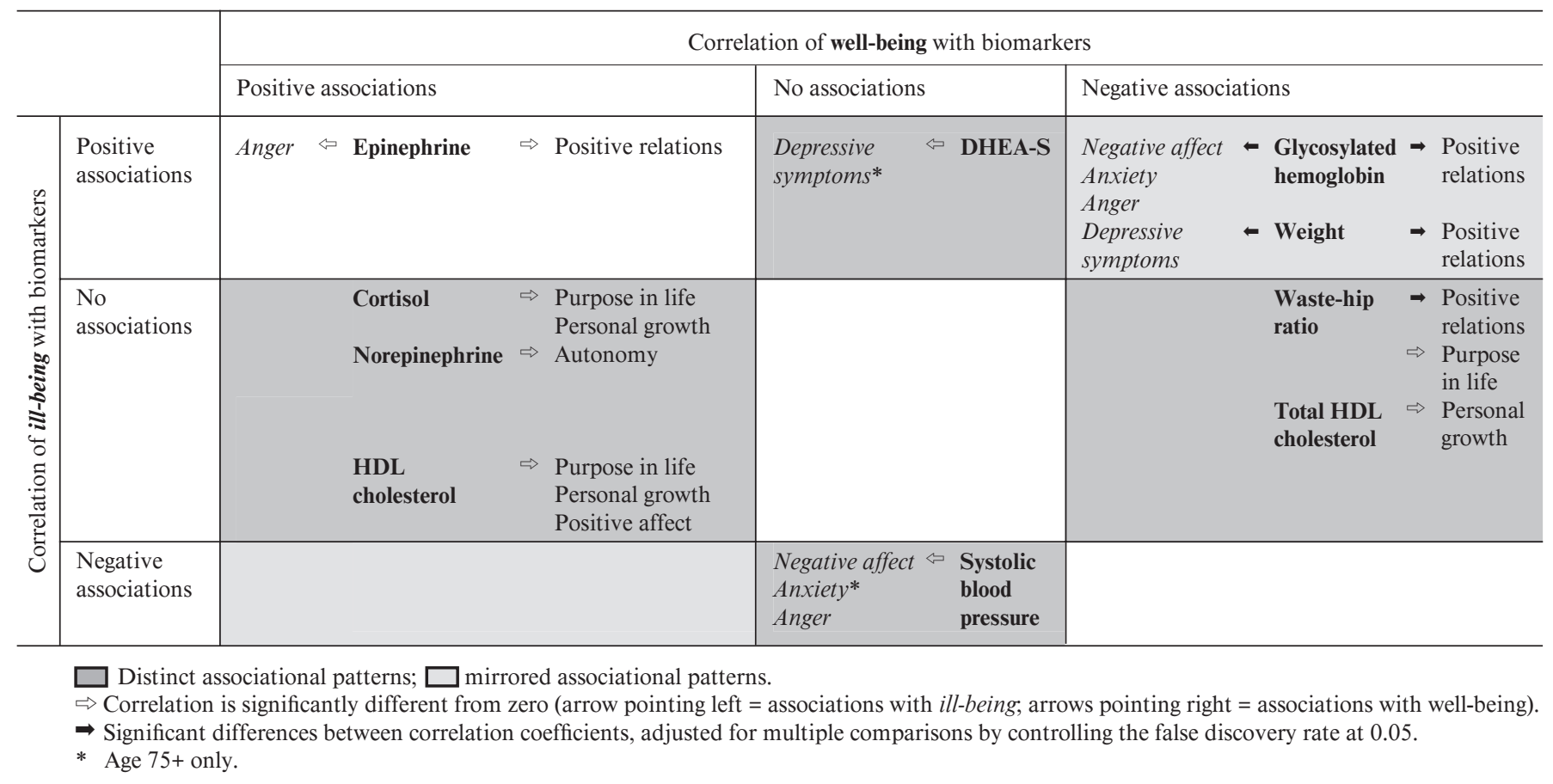

Within each cell, the biomarker for which an effect was obtained is shown in bold. Arrows to the left of the biomarker identify significant associations with ill-being, while arrows to the right identify significant associations with well-being. Open arrows indicate correlations that were significantly different from zero, whereas filled arrows indicate instances in which there was a significant difference between the coefficients for well-being, or illbeing with the same biomarker (adjusting for multiple comparisons using a false discovery rate of 0.05 ).

Seven biomarkers (cortisol, norepinephrine, DHEA-S, waist-hip ratio, systolic blood pressure, HDL cholesterol, total/HDL cholesterol) revealed support for the distinct hypothesis. Three of these showed positive associations with well-being, but not ill-being. Specifically, those with higher levels of life purpose and personal growth had significantly flatter slopes of daily salivary cortisol, while those with higher levels of autonomy had higher levels of norepinephrine. Those with higher levels of purpose in life, personal growth and positive affect also had significantly higher levels of HDL cholesterol. Two other biomarkers showed significant negative associations with well-being, but not ill-being. Specifically, those with higher levels of positive relations and life purpose had lower waist-hip ratios, while those with higher levels of person- al growth had lower total/HDL cholesterol. The remaining two biomarkers showed significant associations with ill-being, but not well-being - those with more depressive symptoms showed higher levels of DHEA-S (full sample and age $75+$ ), while those with higher levels of negative affect, trait anxiety (only 75+) and trait anger had lower levels of systolic blood pressure. With regard to significant differences between correlates of well-being or ill-being with the same biomarker, only the association of positive relations with waist-hip ratio was significantly different from the coefficients of three measures of ill-being (anger, anxiety, negative affect) with the same biomarker.

Two biomarkers (weight, glycosylated hemoglobin) revealed support for the mirrored hypothesis. Those with higher levels of negative affect, trait anxiety and trait anger (full sample and especially those $75+$ ) had significantly higher levels of glycosylated hemoglobin, while those with higher levels of positive relations with others had significantly lower levels of this same biomarker. All of these ill-being and well-being outcomes were also significantly different from each other, as indicated by the bold arrows. Those with higher levels of positive relations with others also had lower weight, while those with greater depressive symptoms had higher weight. These two outcomes were also significantly different from each other. 
The remaining effect in table 3 pertains to epinephrine, which was significantly and positively associated with both trait anger and positive relations with others. This finding conformed to neither the distinct nor mirrored hypothesis.

\section{Discussion}

The purpose of this investigation was to examine the empirical associations between measures of psychological well-being and ill-being with biomarkers, both neuroendocrine and cardiovascular. The findings are limited by the small sample of aging women on which the analyses are based and the cross-sectional design, which offers no insight on causal directionality (does well-being or illbeing influence biology, or does biology influence well-being or ill-being? or are they reciprocally related?). The study is also based on relatively simplistic bivariate analyses. Nonetheless, because few, if any, prior investigations have examined links between measures of positive and negative psychological functioning with biology in the same study, the findings offer a useful place to begin probing basic associations across wide territories of wellbeing, ill-being and biology.

The central question guiding the analyses was whether psychological well-being and ill-being constitute opposite ends of a bipolar continuum, which would predict similar biological correlates with opposite directional patterns (i.e. the mirrored hypothesis), or whether well-being and ill-being are largely independent dimensions of psychological functioning with unique biological correlates (i.e. the distinct hypothesis). The overall pattern of effects was more strongly supportive of the distinct hypothesis. For seven biomarkers, three neuroendocrine (cortisol, DHEA$\mathrm{S}$, norepinephrine) and four cardiovascular (HDL cholesterol, total/HDL cholesterol, systolic blood pressure, waist-hip ratio), significant correlations with well-being (or, ill-being) were not accompanied by significant associations with ill-being (or, well-being, respectively) and the same biomarker. Moreover, of the correlations that reflected the distinct pattern, the majority (69\%) were instances in which measures of well-being were significantly correlated with biomarkers, but no parallel effects were evident for measures of ill-being. Stated another way, psychological well-being showed a more pervasive and distinct biological signature than was evident for psychological ill-being. However, it is important to note that these effects were evident for only a subset of the indicators of eudaimonic well-being (positive relations with oth- ers, purpose in life, personal growth). Surprisingly few significant associations were found for hedonic well-being.

Alternatively, for two biomarkers, namely weight and glycosylated hemoglobin, there was strong evidence of mirrored biological patterns. For both, it was the same measure of well-being (i.e. positive relationships with others) that showed significant differences with the opposite directional pattern obtained for measures of ill-being. Specifically, those with better social relationships had lower weight, while those with higher depressive symptoms had higher weight. Similarly, those with better social relationships had lower levels of glycosylated hemoglobin, while those with higher levels of negative affect, trait anxiety and trait anger had higher levels of the same biomarker. In addition, epinephrine was the single biomarker showing positive associational patterns both with wellbeing (positive relations with others) and ill-being (trait anger).

Although nearly all of the above findings conformed to the anticipated pattern that higher well-being would be associated with lower biological risk, and conversely, that higher ill-being would be associated with higher biological risk, there were select anomalies, the most notable of which was that higher levels of negative affect, anxiety and anger were significantly correlated with lower levels of systolic blood pressure. Whether this is a replicable finding remains to be seen, but we note that a community survey of persons aged 77-99 found that systolic hypotensive individuals had higher levels of negative affect than systolic normotensives [44].

In addition, because the obtained patterns are influenced by sample size and statistical power, important caveats surround any claims that the evidence supports a comparative advantage for the distinct hypothesis. With a larger sample size, some outcomes could well shift from support for the distinct to the mirrored pattern. For example, the positive relationships between slope of daily salivary cortisol and well-being (purpose in life, personal growth) was paralleled by an opposite, but nonsignificant effect of the same biomarker with trait anger. With a sample of $130+$, this effect would have been significant, thereby supporting the mirrored pattern. Alternatively, with a larger sample size, there could also be increased evidence for distinct patterns, such as the overall number of significant effects between markers of HDL cholesterol or total/HDL cholesterol with well-being compared to links between these biomarkers and ill-being, where many notably weak associations were evident. 
However provisional, these findings are nonetheless valuable in adding to the growing literature on how mental maladjustment and well-being are instantiated in biology. Such research increasingly clarifies that what has been learned from prior studies linking depressive symptoms, negative affect, anxiety and anger to various biomarkers, neuroendocrine and cardiovascular [17-27], does not automatically translate to efforts to map the biology of well-being [28-32]. Whether different mechanisms underlie psychological ill-being and well-being is far from clear, but the emerging work does call for greater attention to detailing both the type and level of biomarkers that constitute normal, healthy functioning as distinguished from those that indicate dysregulation.

Three types of inquiry will help move these agendas forward. The first requires population-level assessments to establish distributional characteristics (i.e. norms as to what constitutes low, average and high levels) of both psychological assessments (ill-being and well-being) and biomarkers. Such investigations are critically needed to clarify the generality of the psychobiological linkages across groups differentiated by gender, age, socioeconomic status and race/ethnicity. We note that even with our small sample of women, numerous correlates of well-being with biology (i.e. salivary cortisol) and ill-being with biology (e.g. DHEA-S, systolic blood pressure, glycosylated hemoglobin) were more strongly evident among those aged $75+$, suggesting important life course dynamics in these relationships. Large, representative samples studied longitudinally are also well-suited for tracking downstream health consequences of such mind/body linkages. Which are predictive of subsequent profiles of morbidity and mortality, and which predict maintenance of healthy functioning through time? The juxtaposition of positive and negative mental conditions and how they are instantiated in biology will be central to answering these kinds of questions.

A second venue of future research builds on the distinction between psychological ill-being and well-being, but reminds us that in any given individual, they are combined into some blend of what James [45] referred to as 'healthy-mindedness', on the one hand, and the 'sick soul', on the other. Although the most straightforward combination sees them as inversely linked, there is ample evidence that the absence of mental maladjustment does not guarantee the presence of mental health $[9,10]$, nor does a prior history of mental illness obviate a future of psychological well-being $[13,14]$. Unknown to date is what these differing combinations portend for biology and subsequent health.

Psychological Well-Being and Ill-Being
Finally, the study of life challenges, both in the laboratory and naturalistically, provides an important direction for advancing knowledge of the dynamics of ill-being, well-being and biology. Particularly valuable is the opportunity to study recovery processes. For example, in a driving simulation challenge in the elderly [46] with cortisol assessments prior to the challenge and then every $15 \mathrm{~min}$ for $2.5 \mathrm{~h}$, three categories of response were evident: (a) those showing a rapid initial rise in cortisol followed by a return to prechallenge levels within $45 \mathrm{~min}$ after completing the task; (b) those showing a rapid rise followed by persistently elevated cortisol postchallenge, and (c) those showing no response to the challenge. The first response, regarded as normal, may be predicted by those having high levels of well-being. Indeed, other analyses from these same respondents showed that those with high self-esteem showed this response profile [47]. Alternatively, high levels of ill-being may predict those who show persistently high levels of cortisol postchallenge. The nonresponse group, in turn, may reflect blunted sensitivity of the HPA axis (perhaps linked to prior mental health status), or the fact that high-speed driving simulation was not regarded as challenging.

Outside the laboratory, persistent challenges such as caregiving have also been linked with biological outcomes. A 6-year study of persons caring for a spouse with senile dementia showed increases in inflammatory markers, which were not evident for matched controls without caregiving responsibilities [48]. Caregivers had an average increase in levels of interleukin-6 (IL-6) that was 4 times greater than the increase seen for noncaregivers. These contrasting average profiles might be further illuminated by taking into account ratings of psychological well-being and ill-being, particularly given that the caregiving role has been previously linked with both heightened distress and enhanced well-being [49]. Those who find a deep sense of purpose, meaning and connection in the experience may not show the same cross-time increments in IL-6 that occur for those who find caregiving to be an adverse life stressor. Similarly, among noncaregivers, there may be those with high levels of ill-being who also show marked increments in IL-6 across time. Still, other studies have brought naturally occurring life challenges into the laboratory, for example, by observing online biological responses while couples discuss their marital conflicts [50]. Such studies have shown gender differences in physiological responses to marital conflict (i.e. older husbands exhibited greater cortisol response to conflict discussion, when spousal support was low, compared to wives). But again, this pattern could be sharpened by

Psychother Psychosom 2006;75:85-95 
taking into account the profiles of psychological ill-being or well-being that couples bring with them to these interactions. Those with pre-existing high levels of well-being, both male and female, may show less physiological reactivity to, or quicker recovery from, interaction about issues of conflict, compared with those with high profiles of ill-being.

Elaborating the features of adaptive responses to challenge - what some have referred to as the phenomenon of resilience [51] - will also advance these inquiries. Charney [51] has proposed that the 'resilient profile' is characterized by some of the same biomarkers studied here (i.e. high levels of DHEA-S, low levels of HPA axis activity, $\mathrm{CRH}$ and locus-coeruleus-norepinephrine activity) as well as high levels of neuropeptide $\mathrm{Y}$, galamin, testosterone and $5-\mathrm{HT}_{1 \mathrm{~A}}$ receptor and benzodiazepine receptor function. These constitute promising new directions for mapping the neurophysiological substrates of the resilient, who are also those likely to maintain high levels of psychological well-being in the face of life challenge [52].

\section{Acknowledgement}

This research was supported by the National Institute of Mental Health (P50-MH61083), the National Institute on Aging (P01AG020166) and the National Institutes of Health (M01-RR03186), grant to the GCRC of the University of Wisconsin-Madison.

\section{References}

1 Diener E, Suh EM, Lucas RE, Smith HL: Subjective well-being: three decades of progress. Psychol Bull 1999; 125:276-302.

2 Kahneman D, Diener E, Schwarz N: Well-Being: The Foundations of Hedonic Psychology. New York, Russell Sage Foundation, 1999.

3 Keyes CLM, Shmotkin D, Ryff CD: Optimizing well-being: the empirical encounter of two traditions. J Pers Soc Psychol 2002;82:10071022.

4 Ryan RM, Deci EL: On happiness and human potentials: a review of research on hedonic and eudaimonic well-being. Ann Rev Psychol 2001;52:141-166.

5 Bradburn NM: The Structure of Psychological Well-Being. Chicago, Aldine, 1969.

6 Diener E, Smith H, Fujita F: The personality structure of affect. J Pers Soc Psychol 1995;69: 130-141.

7 Russell JA, Carroll JM: On the bipolarity of positive and negative affect. Psychol Bull 1999; 125:3-30.

8 Yik MSM, Russell JA, Barrett LF: Structure of self-reported current affect: integration and beyond. J Pers Soc Psychol 1999;77:600-619.

$\checkmark 9$ Keyes CLM: The mental health continuum: from languishing to flourishing in life. J Health Soc Behav 2002;43:207-222.

10 Singer BH, Ryff CD, Carr D, Magee WJ: Life histories and mental health: A person-centered strategy; in Raftery A (ed): Sociological Methodology. Washington, American Sociological Association, 1998, pp 1-51.

$\checkmark 11$ Davidson RJ: Affective style and affective disorders: perspectives from affective neuroscience. Cogn Emotion 1998;12:307-330.

-12 Urry HL, Nitschke JB, Dolski I, Jackson DC, Dalton KM, Mueller CJ, Rosenkranz MA, Ryff CD, Singer BH, Davison RJ: Making a life worth living: neural correlates of well-being. Psychol Sci 2004;15:367-372.
13 Fava GA, Rafanelli C, Grandi S, Conti S, Belluardo P: Prevention of recurrent depression with cognitive behavioral therapy. Arch Gen Psychiat 1998;55:816-821.

14 Fava GA, Ruini C, Rafanelli C, Finos L, Conti S, Grandi S: Six-year outcome of cognitive behavior therapy for prevention of recurrent depression. Am J Psychiatry 2004;161:18721876.

15 Ryff CD, Singer BH: The contours of positive human health. Psychol Inq 1998;9:1-28.

16 Ryff CD, Singer BH: Social environments and the genetics of aging: advancing knowledge of protective health mechanisms. J Gerontol Ser B 2005;60:12-23.

17 Brown ES, Varghese FP, McEwen BS: Association of depression with medical illness: Does cortisol play a role? Biol Psychiatry 2004;55: $1-9$.

18 Hughes JW, Watkins L, Blumenthal JA: Depression and anxiety symptoms are related to increased 24-hour urinary norepinephrine excretion among healthy middle-aged women. J Psychosom Res 2004;57:353-358.

19 Polk DE, Cohen S, Doyle WJ: State and trait affect as predictors of salivary cortisol in healthy adults. Psychoneuroendocrinology 2005;30:261-272.

20 Rubin RT, Poland RE, Lesser IM: Neuroendocrine aspects of primary endogenous depression. I. Cortisol secretory dynamics in patients and matched controls. Arch Gen Psychiatry 1987;44:328-336.

21 Barefoot JC, Heitmann BL, Helms MJ, Williams RB, Surwit RS, Siegler IC: Symptoms of depression and changes in body weight from adolescence to mid-life. Int J Obes Relat Metab Disord 1998;22:688-694.

22 Ahlberg AC, Ljung T, Rosmond R: Depression and anxiety symptoms in relation to anthropometry and metabolism in men. Psychiatr Res 2002;112:101-110.
23 Landén M, Baghaei F, Rosmond R: Dyslipidemia and high waist-hip ratio in women with self-reported social anxiety. Psychoneuroendocrinology 2004;29:1037-1046.

24 Schuck P: Glycated hemoglobin as a physiological measure of stress and its relation to some psychological stress indicators. Behav Med 1998;24:89-94.

25 Markovitz JH, Matthews KA, Wing RR, Kuller LH, Meilahn EN: Psychological, biological and health behavior predictors of blood pressure changes in middle-age women. J Hypertens 1991;9:399-406.

-26 Räikkönen R, Matthews KA, Kuller LH, Reiber C, Burher CH: Anger, hostility and visceral adipose tissue in healthy post-menopausal women. Metabolism 1999;489:1146-1151.

27 Steptoe A, Cropley M, Griffith J, Kirschbaum C: Job strain and anger expression predict early morning elevations in salivary cortisol. Psychosom Med 2000;62:286-292.

28 Lindfors P, Lundberg U: Is low cortisol release an indicator of positive health? Stress Health 2002; 18:153-160.

29 Ryff CD, Singer BH, Love GD: Positive health: connecting well-being with biology. Philos Trans R Soc Lond B Biol Sci 2004;359:13831394.

30 Friedman EM, Hayney M, Love GD, Singer BH, Ryff CD: Serum interleukin-6 and soluble IL-6 receptors are modulated by psychological well-being in aging women. Meet Am Psychosom Soc, Vancouver, March, 2005.

31 Uchino BN, Cacioppo JT, Kiecolt-Glaser JK: The relationship between social support and physiological processes: a review with emphasis on underlying mechanisms and implications for health. Psychol Bull 1996;119:488531.
Ryff/Dienberg Love/Urry/Muller/ Rosenkranz/Friedman/Davidson/Singer 
-32 Taylor SE, Lerner JS, Sherman DK, Sage RM, McDowell NK: Are self-enhancing cognitions associated with healthy or unhealthy biological profiles? J Pers Soc Psychol 2003;85:605615.

33 Mroczek DK, Kolarz CM: The effect of age on positive and negative affect: a developmental perspective on happiness. J Pers Soc Psychol 1998;75:1333-1349.

34 Ryff CD, Singer BH: From social structure to biology: integrative science in pursuit of human health and well-being; in Snyder CR, Lopez SJ (eds): Handbook of Positive Psychology. Oxford, Oxford University Press, 2002, pp 541-555.

35 Ryff CD: Happiness is everything, or is it? Explorations on the meaning of psychological well-being. J Pers Soc Psychol 1989;57:10691081.

36 Ryff CD, Keyes CLM: The structure of psychological well-being revisited. J Pers Soc Psychol 1995;69:719-727.

37 Watson D, Clark LA, Tellegen A: Development and validation of brief measures of positive and negative affect: The PANAS scales. J Pers Soc Psychol 1988;54:1063-1070.
Watson D, Clark LA, Weber K, Assenheimer JS, Strauss ME, McCormick RA: Testing a tripartite model. II. Exploring the symptom structure of anxiety and depression in student, adult, and patient samples. J Abnorm Psycho 1995; 104:15-25.

39 Radloff L: The CES-D scale: A self-report depression scale for research in the general population. Appl Psychol Meas 1977;1:385-401.

40 Spielberger CD: Manual for the State-Trait Anxiety Inventory. Palo Alto, Consulting Psychologists Press, 1983.

41 Dixon WJ, Tukey JW: Approximate behavior of the distribution of Winsorized t (Trimming/ Winsorization 2). Technometrics 1968;10:8398.

42 Meng XL, Rosenthal R, Rubin DB: Comparing correlated correlation coefficients. Psychol Bull 1992;111:172-175.

43 Benjamini Y, Hochberg Y: Controlling the false discovery rate: a practical and powerful approach to multiple testing. J Royal Stat Soc B 1995;57:289-300.

44 Jorm A: Association of hypotension with positive and negative affect and depressive symptoms. Br J Psychiatry 2001;178:553-555.

45 James W: The varieties of religious experience. New York, New American Library, 1902.

-46 Seeman TE, Singer B, Charpentier P: Gender differences in HPA axis response to challenge: MacArthur studies of successful aging. Psychoneuroendocrinology 1995;20:711-725.
47 Seeman TE, Berkman LF, Gulanski B, Robbins R, Greenspan S, Rowe J: Response to challenge as a mechanism of successful aging: self-esteem as a predictor of neuroendocrine response. Psychosom Res 1995;39:69-84.

48 Kiecolt-Glaser JK, Preacher KJ, MacCallum RC, Atkinson C, Malarkey WB, Glaser R: Chronic stress and age-related increases in the pro-inflammatory cytokine IL-6. Proc Nat Acad Sci 2003;100:9090-9095.

49 Marks NF: Does it hurt to care? Caregiving, work-family conflict, and midlife well-being. J Marriage Fam 1998;60:951-966.

-50 Heffner KL, Kiecolt-Glaser JK, Loving TJ, Glaser R, Malarkey WB: Spousal support satisfaction as a modifier of physiological responses to marital conflict in younger and older couples. J Behav Med 2004;27:233-254.

51 Charney DS: Psychobiological mechanisms of resilience and vulnerability: implications for successful adaptation to extreme stress. Am J Psychiatry 2004;161:195-216.

52 Ryff $\mathrm{CD}$, Singer BH: Flourishing under fire: resilience as a prototype of challenged thriving; in Keyes CLM, Haidt J (eds): Flourishing: Positive Psychology and the Life Well-Lived. Washington, American Psychological Association, 2003, pp 15-36.

\section{Announcements}

\section{9th Panhellenic Congress of Psychiatry}

Athens, May 4-8, 2006

Congress website: www.era.gr/19 Psychiatric congress

\section{Training Workshop on Well-Being Therapy}

Venice, September 22-23, 2006

Sponsored by the International Federation of Psychotherapy

Faculty: G.A. Fava, C. Belaise, F. Ottolini, C. Ruini

For more information please contact:

Cornelia Erpenbeck

University Hospital Zürich, Department of Psychiatry

Culmannstrasse 8, CH-8091 Zürich (Switzerland)

Tel. +41 4425552 51, Fax +41 4425544 08, E-Mail secretariat@ifp.name 